\title{
Effects of Lactobacillus Reuteri NBF 1 DSM 32203 Supplementation on Healthy Dog Performance
}

\author{
Benedetta Belà ${ }^{*}$, Giulia Pignataro', Roberta Di Prinzio ${ }^{1}$, Daniele Di Simone ${ }^{2}$, Paolo Emidio \\ Crisi $^{1}$ and Alessandro Gramenzi ${ }^{1}$ \\ ${ }^{1}$ Faculty of Veterinary Medicine, University of Teramo, 64100 Piano d'Accio - Teramo, Italy
}

${ }^{2}$ Department of Economics and Finance, University of Bari, Largo Abbazia Santa Scolastica 53, 70124 Bari, Italy

*Corresponding author: Benedetta Belà, Faculty of Veterinary Medicine, University of Teramo, 64100 Piano d'Accio - Teramo, Italy

ARTICLE INFO

Received: 蔧 July 07, 2021

Published: 幽 July 13, 2021

Citation: Benedetta Belà, Giulia Pignataro, Roberta Di Prinzio, Daniele Di Simone, Paolo Emidio Crisi, Alessandro Gramenzi. Effects of Lactobacillus Reuteri NBF 1 DSM 32203 Supplementation on Healthy Dog Performance. Biomed J Sci \& Tech Res 37(1)-2021. BJSTR. MS.ID.005956.

Abbreviations: BW: Bodyweight; BCS: Body Condition Score; CSL: Centro Sperimentale Del Latte; CFU: Colony-Forming Units; FS: Fecal Score
SUMMARY

In this study was evaluated the effectiveness of a specific probiotic strain, $L$. reuteri NBF 1 DSM 32203, on the intestinal health of healthy Golden Retriever adult dogs by analyzing their Bodyweight (BW), Body Condition Score (BCS), fecal quality and fecal moisture. In addition, microbiological analyses were carried out at specific time points during the experiment in order to quantify bacterial species such as E. coli (for the total coliform count) and Lactobacilli. The dogs included in the study were divided into two groups: control group, fed the standard commercial diet with the addition of a placebo, and treated group to which the probiotic was administered. The study lasted 35 days in line with the time needed to assess any effects. BW data showed no differences between the two groups of dogs. The fecal moisture was significantly lower at the end of the trial in the treated group compared with the control group; the beneficial effect of Lactobacillus reuteri NBF 1 DSM 32203 was also confirmed by the values of fecal score recorded among the two groups of dogs. Additionally, at the end of the study period, there was a significant increase of Lactobacilli in the treated group respect to the control group $(\mathrm{P}=<0.001)$. The data collected in this study report the ability of the probiotic strain L. reuteri NBF 1 DSM 32203 to improve fecal quality parameters such as fecal moisture and fecal quality in healthy adult dogs showing an increase in the Lactobacilli count and a little reduction of total coliforms.

\section{Feed Additive Under Test}

The zootechnical feed additive Lactobacillus reuteri NBF 1 DSM 32203 produced by CENTRO SPERIMENTALE DEL LATTE (CSL), is a freeze-dried microbial preparation of Lactobacillus reuteri DSM 32203.

\section{Objective of the Study}

The main objective of the study was to evaluate the impact of the probiotic L. reuteri NBF1 (DSM 32203) on the body weight and fecal quality of healthy adult dogs also evaluating the ability to modulate intestinal microflora by increasing the number of beneficial bacterial species such as lactobacilli and decreasing more pathogenic ones such as E. coli.

\section{Material and Methods \\ Ethical Statement}

The research was conducted according to the directive 2010/63/EU, (article 1 (paragraph 5f) (EUR-Lex - 02010L006320190626 - EN - EUR-Lex (europa.eu)); the study did not imply any form of animal suffering or health risk, since it focused on the administration of a natural substance. However, compliance with the decree ensured a further safeguard for the patient's health (ANNEX_IV_7). The study was carried out under the scientific direction of the Professor Alessandro Gramenzi (University of Teramo, Italy), animal supervisor Dr. Benedetta Belà and the veterinarian in charge Dr. Meri Di Leonardo. 


\section{Animals and Study Design}

The trial took place on $4^{\text {th }}$ October 2020 in the Dietinger Laura breeding, region: Benne Bicocca,3; 10060 - Scalenghe (TO). Healthy adult male and female non pregnant (age $>1$ year) dogs $(\mathrm{n}=40$, Golden Retriever; 19 females, 21 males; $19+21=40$ ) were selected for the study and were randomly assigned to the control group (CTR; $\mathrm{n}=20$; male: female $=9: 11$ ) and to the treated group (LACTO; $=20$; male: female = 3:2); (Tables $1 \mathrm{~A} \& 1 \mathrm{~B}$ ) reports age and weight of each dog included in the study. The design of the practice is a blind trial: the operator on the farm is aware of the animals belonging to the two experimental groups while the operator of the analysis laboratory is not aware of the origin of the sample; in particular, the analyzed dogs were assigned randomly (SurveyMonkey Excel) between the control group and the treated group (as indicated below), thus minimizing selection bias errors. In this way two similar groups were obtained, allowing to better identify the effect of the treatment. Group assignment was organized according to kennel management standard procedure.

Table 1: Age (month), body weight $(\mathrm{Kg})$ and sex of Golden Retriever dogs included in the study: A. Control group, B. Lacto group.

A. Control group

\begin{tabular}{|c|c|c|c|}
\hline Dogs (CTR group) & Age & Body weight & Sex \\
\hline 1 & 27.2 & 32.8 & M \\
\hline 3 & 28.6 & 33.5 & M \\
\hline 4 & 30.2 & 32.8 & M \\
\hline 5 & 40.4 & 34 & M \\
\hline 8 & 42.5 & 32.9 & M \\
\hline 12 & 29.6 & 33.6 & M \\
\hline 13 & 28.4 & 33.8 & M \\
\hline 17 & 30.5 & 32.8 & M \\
\hline 18 & 30.9 & 33.9 & M \\
\hline 19 & 35.6 & 28.5 & $\mathrm{~F}$ \\
\hline 23 & 37.8 & 28.9 & $\mathrm{~F}$ \\
\hline 25 & 40.1 & 30.1 & $\mathrm{~F}$ \\
\hline 27 & 28.9 & 30.4 & $\mathrm{~F}$ \\
\hline 28 & 26.6 & 28.9 & $\mathrm{~F}$ \\
\hline 32 & 31.6 & 28.6 & $\mathrm{~F}$ \\
\hline 33 & 32.5 & 29.6 & $\mathrm{~F}$ \\
\hline 35 & 33.5 & 29.4 & $\mathrm{~F}$ \\
\hline 37 & 34.6 & 29.8 & $\mathrm{~F}$ \\
\hline 39 & 29.7 & 30 & $\mathrm{~F}$ \\
\hline 40 & 30.5 & 28.1 & $\mathrm{~F}$ \\
\hline Average \pm SD & $32.5 \pm 4.6$ & $31.1 \pm 2.2$ & \\
\hline
\end{tabular}

B. Lacto group

\begin{tabular}{|c|c|c|c|}
\hline $\begin{array}{l}\text { Dogs (Lacto } \\
\text { group) }\end{array}$ & Age & Body weight & Sex \\
\hline 2 & 40.1 & 33.4 & M \\
\hline 6 & 28.9 & 33.8 & M \\
\hline 7 & 26.6 & 32.8 & M \\
\hline 9 & 31.6 & 34.5 & M \\
\hline 10 & 32.5 & 32.8 & M \\
\hline 11 & 33.5 & 34.1 & M \\
\hline 14 & 34.6 & 33.9 & M \\
\hline 15 & 29.7 & 33.4 & M \\
\hline 16 & 30.5 & 32.8 & M \\
\hline 20 & 27.8 & 32.8 & M \\
\hline 21 & 26.5 & 33.4 & M \\
\hline 22 & 32.4 & 33.7 & M \\
\hline 24 & 33.5 & 28.6 & $\mathrm{~F}$ \\
\hline 26 & 39.5 & 29.6 & $\mathrm{~F}$ \\
\hline 29 & 34.6 & 29.4 & $\mathrm{~F}$ \\
\hline 30 & 34.8 & 29.8 & $\mathrm{~F}$ \\
\hline 31 & 37.8 & 30 & $\mathrm{~F}$ \\
\hline 34 & 38.7 & 28.1 & $\mathrm{~F}$ \\
\hline 36 & 40.5 & 27.8 & $\mathrm{~F}$ \\
\hline 38 & 42.6 & 27.6 & $\mathrm{~F}$ \\
\hline Average \pm SD & $33.8 \pm 4.8$ & $31.6 \pm 2.4$ & \\
\hline
\end{tabular}

The sample number was calculated using the formula for the comparison between means, fixing an alpha equal to 0.10 and a beta equal to 0.80 ; in this way it results that the minimum number of subjects to analyze is roughly equal to 20 for each group (see statistical analysis paragraph). In order to minimize the bias, the subjects involved in the experiment have been divided in a completely random way between the two groups; in order to carry out the randomisation, each subject was labeled with a unique numeric code and then twenty numeric codes were randomly extracted from those labeled. The subjects with those numeric codes were assigned in the treatment group, the rest in the control group. The control group diet was supplemented by maltodextrin (used as placebo) while, the treated group diet was supplemented with L. reuteri NBF 1 DSM 32203. Cleaning and disinfecting procedures 
of the single fences were carried out and the animals have been individually stabulated. Before starting the trial, an antiparasitic treatment (ecto and enco) was carried out using commercial molecule drugs with no antibacterial effect (Drontal ${ }^{\circledR}$ Plus Flavour Frontline Tri-Act). The dogs were evaluated daily by a veterinarian for any health and welfare concerns throughout the experimental period (two-week acclimation and 35-day study).

\section{Feed Supplement and Diet}

A dry extruded commercial petfood for adult dogs (Table 2) was fed to both the experimental groups, CTR and LACTO, throughout the study; specifically, the commercial diet used in the experiment was the Purina Proplan Large athletic - adult. L. reuteri NBF 1 DSM 32203 as a freeze-dried microbial preparation of L. reuteri NBF 1 DSM 32203, produced by Centro Sperimentale Del Latte (CSL), was added to the LACTO group diet. Dogs were fed a commercial dry pet food once a day based on their maintenance energy requirements (adult dogs: $100 \mathrm{kcal}$ x BW0.67 kg; [1]. and they had free access to potable water as in pets fed dry feed it is recommended to administer water at will and not rationed. Consumption for each dog was measured by weighing the residue before the next day's meal was administered. The dogs were fed once a day and the residue was zero.

Table 2: Diet chemical composition (label).

\begin{tabular}{|c|c|}
\hline Analytical components & Percentage (\%) \\
\hline Moisture (\%) & 9 \\
\hline Crude protein (\%) & 28 \\
\hline Fat (\%) & 18 \\
\hline Fibre (crude) (\%) & 2 \\
\hline ME* $(\mathrm{kcal} / \mathrm{kg})^{*}$ & 3932.7 \\
\hline
\end{tabular}

Note: *ME= Metabolizable energy.

A sample of pet food was analysed at the Laboratory analysis of animal feed, Department of Veterinary medicine, University of Teramo, in order to determine the analytical composition as required by Regulation (EC) No 152/2009 (EUR-Lex - 32009R0152 - EN - EUR-Lex (europa.eu)) (Tables 3A \& 3B) includes the feed analytical composition of both control (CTR) and LACTO group. Dogs belonging to the LACTO group received commercial feed with the addition of $10 \mathrm{~g} / 100 \mathrm{~kg}$ of L. reuteri NBF 1 DSM 32203, corresponding to $5 \times 10^{9}$ Colony-Forming Units (CFU)/kg feed. The process to obtain the right probiotic amount consisted in using $50 \mathrm{~g}$ of the feed additive (standard concentration $\geq 1.0 \times 10^{11}$ $\mathrm{CFU} / \mathrm{g}$ ), pre-mixed in laboratory with $9950 \mathrm{~g}$ of maltodextrins. Then, a total of $20 \mathrm{~g}$ of this pre-mixture was daily added to each $980 \mathrm{~g}$ of commercial feed in the bowl. The CTR group received the commercial diet, with the addition of $20 \mathrm{~g}$ of maltodextrin in $980 \mathrm{~g}$ of dog feed (placebo).
Table 3: Proximate analysis of pet food related to CTR group (A) and LACTO group (B).

Table 3A: Analytical composition of CTR feed.

\begin{tabular}{|c|c|}
\hline Analytical components & Percentage (\%) \\
\hline Humidity \% & 8.35 \\
\hline Dry matter (\%) & 91.65 \\
\hline Crude protein (\%) & 27.69 \\
\hline Crude Fat (\%) & 17.65 \\
\hline Crude Fiber (\%) & 1.82 \\
\hline Crude ash (\%) & 7.56 \\
\hline NFE $(\%)^{*}$ & 36.93 \\
\hline ME** $(\mathrm{kcal} / \mathrm{Kg})$ & 3944.7 \\
\hline
\end{tabular}

Note: ${ }^{*} \mathrm{NFE}=100-$ (humidity + crude protein + crude fat + crude fiber + crude ash).

**Predictive equations (NRC 2006a) for ME in prepared pet foods for dogs and cats (FEDIAF - Nutritional guidelines for complete and complementary pet food for cats and dogs - September 2020).

Table 3B: Analytical composition of LACTO feed.

\begin{tabular}{|c|c|}
\hline Analytical components & Percentage (\%) \\
\hline Humidity \% & 8.42 \\
\hline Dry matter (\%) & 91.58 \\
\hline Crude protein (\%) & 27.56 \\
\hline Crude Fat (\%) & 17.69 \\
\hline Crude Fiber (\%) & 1.84 \\
\hline Crude ash (\%) & 7.49 \\
\hline NFE(\%) & 37 \\
\hline ME** $(\mathrm{kcal} / \mathrm{Kg})^{*}$ & 3944.7 \\
\hline
\end{tabular}

Note: ${ }^{*} \mathrm{NFE}=100-$ (humidity + crude protein + crude fat + crude fiber + crude ash).

**Predictive equations (NRC 2006a) for ME in prepared pet foods for dogs and cats (FEDIAF - Nutritional guidelines for complete and complementary pet food for cats and dogs - September 2020).

Five samples of the feed belonging to the CTR and LACTO group were sent to the Laboratory analysis of animal feed, Department of Veterinary medicine, University of Teramo, in order to verify the number of Lactobacilli in the preparation and the absence of undesired bacteria (Tables $4 \mathrm{~A}-4 \mathrm{~F}$ ) reports sex, body weight and amount of feed given to each dog at the beginning of the study (T0), after 1 week (T1), after 2 weeks (T2), after 3 weeks (T3), after 4 weeks (T4) and at the end of the experiment (T5); the amount of feed to be administered was calculated each time on the basis of the live body weight of the animal monitored weekly. Furthermore, a sample of the LACTO diet was analyzed in order to monitor the concentration of L. reuteri NBF 1 DSM 32203; the results showed that the concentration of the microorganism corresponded to the expectations. 
Table 4: Sex, body weight and amount of feed given to each dog at the beginning of the study (T0; A), after 1 week (T1; B), after 2 weeks (T2; C), after 3 weeks (T3; D), after 4 weeks (T4; E) and at the end of the experiment (T5; F).

Table 4A: Sex, body weight $(\mathrm{Kg})$ and amount of feed given to each dog at the beginning of the study (T0).

\begin{tabular}{|c|c|c|c|c|c|c|}
\hline Dogs (CTR group) & Sex & Body weight & Kg 0.67 & ME* Kcal/d & ME* Kcal/Kg feed & g/d \\
\hline 1 & M & 32.8 & 13.71 & 1507.64 & 3944.7 & 382 \\
\hline 3 & M & 33.5 & 13.92 & 1531.71 & 3944.7 & 388 \\
\hline 4 & M & 32.8 & 13.71 & 1507.64 & 3944.7 & 382 \\
\hline 5 & $\mathrm{M}$ & 34 & 14.08 & 1548.82 & 3944.7 & 393 \\
\hline 8 & M & 32.9 & 13.74 & 1511.09 & 3944.7 & 383 \\
\hline 12 & M & 33.6 & 13.96 & 1535.14 & 3944.7 & 389 \\
\hline 13 & M & 33.8 & 14.02 & 1541.99 & 3944.7 & 391 \\
\hline 17 & M & 32.8 & 13.71 & 1507.64 & 3944.7 & 382 \\
\hline 18 & M & 33.9 & 14.05 & 1545.41 & 3944.7 & 392 \\
\hline 19 & $\mathrm{~F}$ & 28.5 & 12.33 & 1356.83 & 3944.7 & 344 \\
\hline 23 & $\mathrm{~F}$ & 28.9 & 12.46 & 1371.09 & 3944.7 & 348 \\
\hline 25 & $\mathrm{~F}$ & 30.1 & 12.85 & 1413.57 & 3944.7 & 358 \\
\hline 27 & $\mathrm{~F}$ & 30.4 & 12.95 & 1424.12 & 3944.7 & 361 \\
\hline 28 & $\mathrm{~F}$ & 28.9 & 12.46 & 1371.09 & 3944.7 & 348 \\
\hline 32 & $\mathrm{~F}$ & 28.6 & 12.37 & 1360.4 & 3944.7 & 345 \\
\hline 33 & $\mathrm{~F}$ & 29.6 & 12.69 & 1395.92 & 3944.7 & 354 \\
\hline 35 & $\mathrm{~F}$ & 29.4 & 12.63 & 1388.84 & 3944.7 & 352 \\
\hline 37 & $\mathrm{~F}$ & 29.8 & 12.82 & 1402.99 & 3944.7 & 356 \\
\hline 39 & $\mathrm{~F}$ & 30 & 12.75 & 1410.05 & 3944.7 & 357 \\
\hline 40 & $\mathrm{~F}$ & 28.1 & 12.2 & 1342.53 & 3944.7 & 340 \\
\hline Dogs (LACTO group) & Sex & Body weight & Kg 0.67 & ME* Kcal/d & ME* Kcal/Kg feed & g/d \\
\hline 2 & $\mathrm{M}$ & 33.4 & 13.89 & 1528.28 & 3944.7 & 387 \\
\hline 6 & M & 33.8 & 14.02 & 1541.99 & 3944.7 & 391 \\
\hline 7 & M & 32.8 & 13.71 & 1507.64 & 3944.7 & 382 \\
\hline 9 & M & 34.5 & 14.24 & 1565.87 & 3944.7 & 397 \\
\hline 10 & M & 32.8 & 13.71 & 1507.64 & 3944.7 & 382 \\
\hline 11 & M & 34.1 & 14.11 & 1552.24 & 3944.7 & 393 \\
\hline 14 & M & 33.9 & 14.05 & 1545.41 & 3944.7 & 392 \\
\hline 15 & M & 33.4 & 13.89 & 1528.28 & 3944.7 & 387 \\
\hline 16 & M & 32.8 & 13.71 & 1507.64 & 3944.7 & 382 \\
\hline 20 & M & 32.8 & 13.71 & 1507.64 & 3944.7 & 382 \\
\hline 21 & $\mathrm{M}$ & 33.4 & 13.89 & 1528.28 & 3944.7 & 387 \\
\hline 22 & M & 33.7 & 13.99 & 1538.56 & 3944.7 & 390 \\
\hline 24 & $\mathrm{~F}$ & 28.6 & 12.37 & 1360.4 & 3944.7 & 345 \\
\hline 26 & $\mathrm{~F}$ & 29.6 & 12.69 & 1395.92 & 3944.7 & 354 \\
\hline 29 & $\mathrm{~F}$ & 29.4 & 12.63 & 1388.84 & 3944.7 & 352 \\
\hline 30 & $\mathrm{~F}$ & 29.8 & 12.75 & 1402.99 & 3944.7 & 356 \\
\hline 31 & $\mathrm{~F}$ & 30 & 12.82 & 1410.05 & 3944.7 & 357 \\
\hline 34 & $\mathrm{~F}$ & 28.1 & 12.2 & 1342.53 & 3944.7 & 340 \\
\hline 36 & $\mathrm{~F}$ & 27.8 & 12.11 & 1331.76 & 3944.7 & 338 \\
\hline 38 & $\mathrm{~F}$ & 27.6 & 12.04 & 1324.57 & 3944.7 & 336 \\
\hline
\end{tabular}

Note: *ME $=$ Metabolizable energy 
Table 4B: Sex, body weight (Kg) and amount of feed given to each dog after 1 week of study (T1).

\begin{tabular}{|c|c|c|c|c|c|c|}
\hline Dogs (CTR group) & Sex & Body weight & Kg 0.67 & ME* Kcal/d & ME* Kcal/Kg feed & $g / d$ \\
\hline 1 & M & 32.7 & 13.67 & 1504,19 & 3944.7 & 381 \\
\hline 3 & M & 33.4 & 13.89 & 1528.28 & 3944.7 & 387 \\
\hline 4 & M & 32.9 & 13.74 & 1511.09 & 3944.7 & 383 \\
\hline 5 & M & 34 & 14.08 & 1548.82 & 3944.7 & 393 \\
\hline 8 & M & 32.9 & 13.74 & 1511.09 & 3944.7 & 383 \\
\hline 12 & M & 33.4 & 13.89 & 1528.28 & 3944.7 & 387 \\
\hline 13 & M & 33.7 & 13.99 & 1538.56 & 3944.7 & 390 \\
\hline 17 & M & 32.7 & 13.67 & 1504.19 & 3944.7 & 381 \\
\hline 18 & M & 33.8 & 14.02 & 1541.99 & 3944.7 & 391 \\
\hline 19 & $\mathrm{~F}$ & 28.7 & 12.4 & 1363.97 & 3944.7 & 346 \\
\hline 23 & $\mathrm{~F}$ & 29 & 12.5 & 1374.65 & 3944.7 & 348 \\
\hline 25 & $\mathrm{~F}$ & 30 & 12.82 & 1410.05 & 3944.7 & 357 \\
\hline 27 & $\mathrm{~F}$ & 30.3 & 12.91 & 1420.61 & 3944.7 & 360 \\
\hline 28 & $\mathrm{~F}$ & 28.9 & 12.46 & 1371.09 & 3944.7 & 348 \\
\hline 32 & $\mathrm{~F}$ & 28.7 & 12.4 & 1363.97 & 3944.7 & 346 \\
\hline 33 & $\mathrm{~F}$ & 29.7 & 12.72 & 1399.46 & 3944.7 & 355 \\
\hline 35 & $\mathrm{~F}$ & 29.3 & 12.59 & 1385.3 & 3944.7 & 351 \\
\hline 37 & $\mathrm{~F}$ & 29.6 & 12.69 & 1395.92 & 3944.7 & 354 \\
\hline 39 & $\mathrm{~F}$ & 29.9 & 12.79 & 1406.52 & 3944.7 & 357 \\
\hline 40 & $\mathrm{~F}$ & 28.2 & 12.24 & 1346.11 & 3944.7 & 341 \\
\hline Dogs (LACTO group) & Sex & Body weight & Kg 0.67 & ME* Kcal/d & ME* Kcal/Kg feed & $\mathrm{g} / \mathrm{d}$ \\
\hline 2 & M & 33.3 & 13.86 & 1524.85 & 3944.7 & 387 \\
\hline 6 & M & 33.8 & 14.02 & 1541.99 & 3944.7 & 391 \\
\hline 7 & M & 32.7 & 13.67 & 1504.19 & 3944.7 & 381 \\
\hline 9 & M & 34.4 & 14.2 & 1562.47 & 3944.7 & 396 \\
\hline 10 & M & 32.9 & 13.74 & 1511.09 & 3944.7 & 383 \\
\hline 11 & M & 34.1 & 14.11 & 1552.24 & 3944.7 & 393 \\
\hline 14 & M & 33.9 & 14.05 & 1545.41 & 3944.7 & 392 \\
\hline 15 & M & 33.4 & 13.89 & 1528.28 & 3944.7 & 387 \\
\hline 16 & M & 32.7 & 13.67 & 1504.19 & 3944.7 & 381 \\
\hline 20 & M & 32.6 & 13.64 & 1500.74 & 3944.7 & 380 \\
\hline 21 & M & 33.4 & 13.89 & 1528.28 & 3944.7 & 387 \\
\hline 22 & M & 33.7 & 13.99 & 1538.56 & 3944.7 & 390 \\
\hline 24 & $\mathrm{~F}$ & 28.7 & 12.4 & 1363.97 & 3944.7 & 346 \\
\hline 26 & $\mathrm{~F}$ & 29.7 & 12.72 & 1399.46 & 3944.7 & 355 \\
\hline 29 & $\mathrm{~F}$ & 29.3 & 12.59 & 1385.3 & 3944.7 & 351 \\
\hline 30 & $\mathrm{~F}$ & 29.9 & 12.79 & 1406.52 & 3944.7 & 357 \\
\hline 31 & $\mathrm{~F}$ & 30 & 12.82 & 1410.05 & 3944.7 & 357 \\
\hline 34 & $\mathrm{~F}$ & 28.1 & 12.2 & 1342.53 & 3944.7 & 340 \\
\hline 36 & F & 27.7 & 12.07 & 1328.17 & 3944.7 & 337 \\
\hline 38 & F & 27.7 & 12.07 & 1328.17 & 3944.7 & 337 \\
\hline
\end{tabular}

Note: *ME = Metabolizable energy 
Table 4C: Sex, body weight (Kg) and amount of feed given to each dog after 2 weeks of study (T2).

\begin{tabular}{|c|c|c|c|c|c|c|}
\hline Dogs (CTR group) & Sex & Body weight & Kg 0.67 & ME* Kcal/d & ME* Kcal/Kg feed & $g / d$ \\
\hline 1 & M & 32.7 & 13.67 & 1504.19 & 3944.7 & 381 \\
\hline 3 & M & 33.5 & 13.92 & 1531.71 & 3944.7 & 388 \\
\hline 4 & M & 32.8 & 13.71 & 1507.64 & 3944.7 & 382 \\
\hline 5 & M & 34.1 & 14.11 & 1552.24 & 3944.7 & 393 \\
\hline 8 & M & 32.8 & 13.71 & 1507.64 & 3944.7 & 382 \\
\hline 12 & M & 33.3 & 13.86 & 1524.85 & 3944.7 & 387 \\
\hline 13 & M & 33.7 & 13.99 & 1538.56 & 3944.7 & 390 \\
\hline 17 & M & 32.6 & 13.64 & 1500.74 & 3944.7 & 380 \\
\hline 18 & M & 33.7 & 13.99 & 1538.56 & 3944.7 & 390 \\
\hline 19 & $\mathrm{~F}$ & 28.6 & 12.37 & 1360.4 & 3944.7 & 345 \\
\hline 23 & $\mathrm{~F}$ & 28.9 & 12.46 & 1371.09 & 3944.7 & 348 \\
\hline 25 & $\mathrm{~F}$ & 30.2 & 12.88 & 1417.09 & 3944.7 & 359 \\
\hline 27 & $\mathrm{~F}$ & 30.4 & 12.95 & 1424.12 & 3944.7 & 361 \\
\hline 28 & F & 28.9 & 12.46 & 1371.09 & 3944.7 & 348 \\
\hline 32 & $\mathrm{~F}$ & 28.8 & 12.43 & 1367.53 & 3944.7 & 347 \\
\hline 33 & $\mathrm{~F}$ & 29.6 & 12.69 & 1395.92 & 3944.7 & 354 \\
\hline 35 & $\mathrm{~F}$ & 29.3 & 12.59 & 1385.3 & 3944.7 & 351 \\
\hline 37 & $\mathrm{~F}$ & 29.7 & 12.72 & 1399.46 & 3944.7 & 355 \\
\hline 39 & $\mathrm{~F}$ & 30 & 12.82 & 1410.05 & 3944.7 & 357 \\
\hline 40 & $\mathrm{~F}$ & 28.3 & 12.27 & 1349.69 & 3944.7 & 342 \\
\hline Dogs (LACTO group) & Sex & Body weight & Kg 0.67 & ME* Kcal/d & ME* Kcal/Kg feed & g/d \\
\hline 2 & M & 33.3 & 13.86 & 1524.85 & 3944.7 & 387 \\
\hline 6 & M & 33.7 & 13.99 & 1538.56 & 3944.7 & 390 \\
\hline 7 & M & 32.6 & 13.64 & 1500.74 & 3944.7 & 380 \\
\hline 9 & M & 34.3 & 14.17 & 1559.06 & 3944.7 & 395 \\
\hline 10 & $\mathrm{M}$ & 33 & 13.77 & 1514.53 & 3944.7 & 384 \\
\hline 11 & M & 34.1 & 14.11 & 1552.24 & 3944.7 & 393 \\
\hline 14 & M & 33.9 & 14.05 & 1545.41 & 3944.7 & 392 \\
\hline 15 & M & 33.5 & 13.92 & 1531.71 & 3944.7 & 388 \\
\hline 16 & M & 32.6 & 13.64 & 1500.74 & 3944.7 & 380 \\
\hline 20 & M & 32.7 & 13.67 & 1504.19 & 3944.7 & 381 \\
\hline 21 & M & 33.4 & 13.89 & 1528.28 & 3944.7 & 387 \\
\hline 22 & M & 33.7 & 13.99 & 1538.56 & 3944.7 & 390 \\
\hline 24 & $\mathrm{~F}$ & 28.8 & 12.43 & 1367.53 & 3944.7 & 347 \\
\hline 26 & $\mathrm{~F}$ & 29.7 & 12.72 & 1399.46 & 3944.7 & 355 \\
\hline 29 & $\mathrm{~F}$ & 29.4 & 12.63 & 1388.84 & 3944.7 & 352 \\
\hline 30 & $\mathrm{~F}$ & 29.8 & 12.75 & 1402.99 & 3944.7 & 356 \\
\hline 31 & $\mathrm{~F}$ & 30.1 & 12.85 & 1413.57 & 3944.7 & 358 \\
\hline 34 & $\mathrm{~F}$ & 28.2 & 12.24 & 1346.11 & 3944.7 & 341 \\
\hline 36 & F & 27.8 & 12.11 & 1331.76 & 3944.7 & 338 \\
\hline 38 & F & 27.7 & 12.07 & 1328.17 & 3944.7 & 337 \\
\hline
\end{tabular}

Note: *ME $=$ Metabolizable energy 
Table 4D: Sex, body weight $(\mathrm{Kg})$ and amount of feed given to each dog after 3 weeks of study (T3).

\begin{tabular}{|c|c|c|c|c|c|c|}
\hline Dogs (CTR group) & Sex & Body weight & Kg 0.67 & $\mathrm{ME}^{*} \mathrm{Kcal} / \mathrm{d}$ & $\mathrm{ME}^{*} \mathrm{Kcal} / \mathrm{Kg}$ feed & $g / d$ \\
\hline 1 & M & 32.6 & 13.64 & 1500.74 & 3944.7 & 380 \\
\hline 3 & M & 33.4 & 13.89 & 1528.28 & 3944.7 & 387 \\
\hline 4 & M & 32.9 & 13.74 & 1511.09 & 3944.7 & 383 \\
\hline 5 & M & 34.2 & 14.14 & 1555.65 & 3944.7 & 394 \\
\hline 8 & M & 32.8 & 13.71 & 1507.64 & 3944.7 & 382 \\
\hline 12 & M & 33.3 & 13.86 & 1524.85 & 3944.7 & 387 \\
\hline 13 & M & 33.8 & 14.02 & 1541.99 & 3944.7 & 391 \\
\hline 17 & M & 32.7 & 13.67 & 1504.19 & 3944.7 & 381 \\
\hline 18 & M & 33.8 & 14.02 & 1541.99 & 3944.7 & 391 \\
\hline 19 & $\mathrm{~F}$ & 28.7 & 12.4 & 1363.97 & 3944.7 & 346 \\
\hline 23 & $\mathrm{~F}$ & 28.8 & 12.43 & 1367.53 & 3944.7 & 347 \\
\hline 25 & $\mathrm{~F}$ & 30.1 & 12.85 & 1413.57 & 3944.7 & 358 \\
\hline 27 & $\mathrm{~F}$ & 30.3 & 12.91 & 1420.61 & 3944.7 & 360 \\
\hline 28 & $\mathrm{~F}$ & 28.8 & 12.43 & 1367.53 & 3944.7 & 347 \\
\hline 32 & $\mathrm{~F}$ & 28.7 & 12.4 & 1363.97 & 3944.7 & 346 \\
\hline 33 & $\mathrm{~F}$ & 29.8 & 12.75 & 1402.99 & 3944.7 & 356 \\
\hline 35 & $\mathrm{~F}$ & 29.4 & 12.63 & 1388.84 & 3944.7 & 352 \\
\hline 37 & $\mathrm{~F}$ & 29.8 & 12.75 & 1402.99 & 3944.7 & 356 \\
\hline 39 & $\mathrm{~F}$ & 30.1 & 12.85 & 1413.57 & 3944.7 & 358 \\
\hline 40 & $\mathrm{~F}$ & 28.4 & 12.3 & 1353.26 & 3944.7 & 343 \\
\hline Dogs (LACTO group) & Sex & Body weight & Kg 0.67 & ME* Kcal/d & ME* Kcal/Kg feed & $g / d$ \\
\hline 2 & M & 33.2 & 13.83 & 1521.41 & 3944.7 & 386 \\
\hline 6 & M & 33.6 & 13.96 & 1535.14 & 3944.7 & 389 \\
\hline 7 & M & 32.6 & 13.64 & 1500.74 & 3944.7 & 380 \\
\hline 9 & M & 34.4 & 14.2 & 1562.47 & 3944.7 & 396 \\
\hline 10 & M & 32.9 & 13.74 & 1511.09 & 3944.7 & 383 \\
\hline 11 & M & 34.1 & 14.11 & 1552.24 & 3944.7 & 393 \\
\hline 14 & M & 33.8 & 14.02 & 1541.99 & 3944.7 & 391 \\
\hline 15 & M & 33.5 & 13.92 & 1531.71 & 3944.7 & 388 \\
\hline 16 & M & 32.6 & 13.64 & 1500.74 & 3944.7 & 380 \\
\hline 20 & M & 32.8 & 13.71 & 1507.64 & 3944.7 & 382 \\
\hline 21 & M & 33.3 & 13.86 & 1524.85 & 3944.7 & 387 \\
\hline 22 & M & 33.6 & 13.96 & 1535.14 & 3944.7 & 389 \\
\hline 24 & $\mathrm{~F}$ & 28.7 & 12.4 & 1363.97 & 3944.7 & 346 \\
\hline 26 & $\mathrm{~F}$ & 29.6 & 12.69 & 1395.92 & 3944.7 & 354 \\
\hline 29 & $\mathrm{~F}$ & 29.3 & 12.59 & 1385.3 & 3944.7 & 351 \\
\hline 30 & $\mathrm{~F}$ & 29.9 & 12.79 & 1406.52 & 3944.7 & 357 \\
\hline 31 & $\mathrm{~F}$ & 30.2 & 12.88 & 1417.09 & 3944.7 & 359 \\
\hline 34 & $\mathrm{~F}$ & 28.3 & 12.27 & 1349.69 & 3944.7 & 342 \\
\hline 36 & $\mathrm{~F}$ & 27.7 & 12.07 & 1328.17 & 3944.7 & 337 \\
\hline 38 & $\mathrm{~F}$ & 27.6 & 12.04 & 1324.57 & 3944.7 & 336 \\
\hline
\end{tabular}

Note: *ME = Metabolizable energy 
Table 4E: Sex, body weight $(\mathrm{Kg})$ and amount of feed given to each dog after 4 weeks of study (T4).

\begin{tabular}{|c|c|c|c|c|c|c|}
\hline Dogs (CTR group) & Sex & Body weight & Kg 0.67 & ME* Kcal/d & ME* Kcal/Kg feed & $g / d$ \\
\hline 1 & M & 32.7 & 13.67 & 1504.19 & 3944.7 & 381 \\
\hline 3 & M & 33.3 & 13.86 & 1524.85 & 3944.7 & 387 \\
\hline 4 & M & 33 & 13.77 & 1514.53 & 3944.7 & 384 \\
\hline 5 & M & 34.1 & 14.11 & 1552.24 & 3944.7 & 393 \\
\hline 8 & M & 32.8 & 13.71 & 1507.64 & 3944.7 & 382 \\
\hline 12 & M & 33.5 & 13.92 & 1531.71 & 3944.7 & 388 \\
\hline 13 & M & 33.7 & 13.99 & 1538.56 & 3944.7 & 390 \\
\hline 17 & M & 32.8 & 13.71 & 1507.64 & 3944.7 & 382 \\
\hline 18 & M & 33.9 & 14.05 & 1545.41 & 3944.7 & 392 \\
\hline 19 & $\mathrm{~F}$ & 28.8 & 12.43 & 1367.53 & 3944.7 & 347 \\
\hline 23 & $\mathrm{~F}$ & 28.8 & 12.43 & 1367.53 & 3944.7 & 347 \\
\hline 25 & $\mathrm{~F}$ & 30 & 12.82 & 1410.05 & 3944.7 & 357 \\
\hline 27 & $\mathrm{~F}$ & 30.3 & 12.91 & 1420.61 & 3944.7 & 360 \\
\hline 28 & $\mathrm{~F}$ & 28.8 & 12.43 & 1367.53 & 3944.7 & 347 \\
\hline 32 & $\mathrm{~F}$ & 28.8 & 12.43 & 1367.53 & 3944.7 & 347 \\
\hline 33 & $\mathrm{~F}$ & 29.7 & 12.72 & 1399.46 & 3944.7 & 355 \\
\hline 35 & $\mathrm{~F}$ & 29.4 & 12.63 & 1388.84 & 3944.7 & 352 \\
\hline 37 & $\mathrm{~F}$ & 29.8 & 12.75 & 1402.99 & 3944.7 & 356 \\
\hline 39 & $\mathrm{~F}$ & 30 & 12.82 & 1410.05 & 3944.7 & 357 \\
\hline 40 & $\mathrm{~F}$ & 28.2 & 12.24 & 1346.11 & 3944.7 & 341 \\
\hline Dogs (LACTO group) & Sex & Body weight & Kg 0.67 & $\mathrm{ME}^{*} \mathrm{Kcal} / \mathrm{d}$ & ME* Kcal/Kg feed & $g / d$ \\
\hline 2 & M & 33.3 & 13.86 & 1524.85 & 3944.7 & 387 \\
\hline 6 & M & 33.7 & 13.99 & 1538.56 & 3944.7 & 390 \\
\hline 7 & M & 32.6 & 13.64 & 1500.74 & 3944.7 & 380 \\
\hline 9 & M & 34.5 & 14.24 & 1565.87 & 3944.7 & 397 \\
\hline 10 & M & 32.9 & 13.74 & 1511.09 & 3944.7 & 383 \\
\hline 11 & M & 34.1 & 14.11 & 1552.24 & 3944.7 & 393 \\
\hline 14 & M & 33.7 & 13.99 & 1538.56 & 3944.7 & 390 \\
\hline 15 & M & 33.5 & 13.92 & 1531.71 & 3944.7 & 388 \\
\hline 16 & M & 32.6 & 13.64 & 1500.74 & 3944.7 & 380 \\
\hline 20 & M & 32.8 & 13.71 & 1507.64 & 3944.7 & 382 \\
\hline 21 & $\mathrm{M}$ & 33.3 & 13.86 & 1524.85 & 3944.7 & 387 \\
\hline 22 & M & 33.6 & 13.96 & 1535.14 & 3944.7 & 389 \\
\hline 24 & $\mathrm{~F}$ & 28.6 & 12.37 & 1360.4 & 3944.7 & 345 \\
\hline 26 & $\mathrm{~F}$ & 29.6 & 12.69 & 1395.92 & 3944.7 & 354 \\
\hline 29 & $\mathrm{~F}$ & 29.4 & 12.63 & 1388.84 & 3944.7 & 352 \\
\hline 30 & $\mathrm{~F}$ & 29.8 & 12.75 & 1402.99 & 3944.7 & 356 \\
\hline 31 & $\mathrm{~F}$ & 30.2 & 12.88 & 1417.09 & 3944.7 & 359 \\
\hline 34 & $\mathrm{~F}$ & 28.2 & 12.24 & 1346.11 & 3944.7 & 341 \\
\hline 36 & $\mathrm{~F}$ & 27.8 & 12.11 & 1331.76 & 3944.7 & 338 \\
\hline 38 & $\mathrm{~F}$ & 27.7 & 12.07 & 1328.17 & 3944.7 & 337 \\
\hline
\end{tabular}

Note: *ME = Metabolizable energy 
Table 4F: Sex, body weight $(\mathrm{Kg})$ and amount of feed given to each dog at the end of the study (T5).

\begin{tabular}{|c|c|c|c|c|c|c|}
\hline Dogs (CTR group) & Sex & Body weight & Kg 0.67 & $\mathrm{ME}^{*} \mathrm{Kcal} / \mathrm{d}$ & ME* Kcal/Kg feed & $\mathrm{g} / \mathrm{d}$ \\
\hline 1 & M & 32.8 & 13.71 & 1507.64 & 3944.7 & 382 \\
\hline 3 & M & 33.4 & 13.89 & 1528.28 & 3944.7 & 387 \\
\hline 4 & M & 32.8 & 13.71 & 1507.64 & 3944.7 & 382 \\
\hline 5 & M & 34 & 14.08 & 1548.82 & 3944.7 & 393 \\
\hline 8 & M & 32.7 & 13.67 & 1504.19 & 3944.7 & 381 \\
\hline 12 & M & 33.5 & 13.92 & 1531.71 & 3944.7 & 388 \\
\hline 13 & M & 33.6 & 13.96 & 1535.14 & 3944.7 & 389 \\
\hline 17 & M & 32.9 & 13.74 & 1511.09 & 3944.7 & 383 \\
\hline 18 & M & 34 & 14.08 & 1548.82 & 3944.7 & 393 \\
\hline 19 & $\mathrm{~F}$ & 28.7 & 12.4 & 1363.97 & 3944.7 & 346 \\
\hline 23 & $\mathrm{~F}$ & 28.8 & 12.43 & 1367.53 & 3944.7 & 347 \\
\hline 25 & $\mathrm{~F}$ & 30 & 12.82 & 1410.05 & 3944.7 & 357 \\
\hline 27 & $\mathrm{~F}$ & 30.2 & 12.88 & 1417.09 & 3944.7 & 359 \\
\hline 28 & $\mathrm{~F}$ & 28.8 & 12.43 & 1367.53 & 3944.7 & 347 \\
\hline 32 & $\mathrm{~F}$ & 28.6 & 12.37 & 1360.4 & 3944.7 & 345 \\
\hline 33 & $\mathrm{~F}$ & 29.7 & 12.72 & 1399.46 & 3944.7 & 355 \\
\hline 35 & $\mathrm{~F}$ & 29.5 & 12.66 & 1392.38 & 3944.7 & 353 \\
\hline 37 & $\mathrm{~F}$ & 29.9 & 12.79 & 1406.52 & 3944.7 & 357 \\
\hline 39 & $\mathrm{~F}$ & 30 & 12.82 & 1410.05 & 3944.7 & 357 \\
\hline 40 & $\mathrm{~F}$ & 28.3 & 12.27 & 1349.69 & 3944.7 & 342 \\
\hline Dogs (LACTO group) & Sex & Body weight & Kg 0.67 & $\mathrm{ME}^{*} \mathrm{Kcal} / \mathrm{d}$ & ME* Kcal/Kg feed & $g / d$ \\
\hline 2 & M & 33.4 & 13.89 & 1528.28 & 3944.7 & 387 \\
\hline 6 & M & 33.8 & 14.02 & 1541.99 & 3944.7 & 391 \\
\hline 7 & M & 32.7 & 13.67 & 1504.19 & 3944.7 & 381 \\
\hline 9 & M & 34.4 & 14.2 & 1562.47 & 3944.7 & 396 \\
\hline 10 & M & 32.9 & 13.74 & 1511.09 & 3944.7 & 383 \\
\hline 11 & M & 34.1 & 14.11 & 1552.24 & 3944.7 & 393 \\
\hline 14 & M & 33.8 & 14.02 & 1541.99 & 3944.7 & 391 \\
\hline 15 & M & 33.6 & 13.96 & 1535.14 & 3944.7 & 389 \\
\hline 16 & M & 32.7 & 13.67 & 1504.19 & 3944.7 & 381 \\
\hline 20 & M & 32.9 & 13.74 & 1511.09 & 3944.7 & 383 \\
\hline 21 & M & 33.3 & 13.86 & 1524.85 & 3944.7 & 387 \\
\hline 22 & M & 33.5 & 13.92 & 1531.71 & 3944.7 & 388 \\
\hline 24 & $\mathrm{~F}$ & 28.7 & 12.4 & 1363.97 & 3944.7 & 346 \\
\hline 26 & $\mathrm{~F}$ & 29.6 & 12.69 & 1395.92 & 3944.7 & 354 \\
\hline 29 & $\mathrm{~F}$ & 29.3 & 12.59 & 1385.3 & 3944.7 & 351 \\
\hline 30 & $\mathrm{~F}$ & 29.9 & 12.79 & 1406.52 & 3944.7 & 357 \\
\hline 31 & $\mathrm{~F}$ & 30.1 & 12.85 & 1413.57 & 3944.7 & 358 \\
\hline 34 & $\mathrm{~F}$ & 28.1 & 12.2 & 1342.53 & 3944.7 & 340 \\
\hline 36 & $\mathrm{~F}$ & 27.9 & 12.14 & 1335.35 & 3944.7 & 339 \\
\hline 38 & $\mathrm{~F}$ & 27.7 & 12.07 & 1328.17 & 3944.7 & 337 \\
\hline
\end{tabular}

Note: *ME = Metabolizable energy 
a) Diet Composition: Chicken (19\%), Wheat, Dehydrated Poultry Protein, Corn, Rice (8\%), Animal Fat, Corn Gluten Flour, Dehydrated Beet Pulp, Corn Semola, Wheat Gluten Flour, Soybeans, Salmon Dehydrated Protein, Minerals, Dehydrated Eggs, Fish Oil.

b) Nutritional Additives per Kg: Vitamin A: $29000 \mathrm{IU} /$ $\mathrm{kg}$, Vitamin D3: $950 \mathrm{IU} / \mathrm{kg}$, Vitamin E: $550 \mathrm{IU} / \mathrm{kg}$, Vitamin C: $140 \mathrm{mg} / \mathrm{kg}$, ferrous sulfate monohydrate: $240 \mathrm{mg} / \mathrm{kg}$, anhydrous calcium iodate: $3.1 \mathrm{mg} / \mathrm{kg}$, copper sulfate pentahydrate: $48 \mathrm{mg} /$ $\mathrm{kg}$, manganose sulfate monohydrate: $110 \mathrm{mg} / \mathrm{kg}$, zinc sulphate monohydrate: $400 \mathrm{mg} / \mathrm{kg}$, sodium selenite: $0.28 \mathrm{mg} / \mathrm{kg}$, tocopherol extracts from vegetable oils: $50 \mathrm{mg} / \mathrm{kg}$.

\section{Data Collection}

Bodyweight (BW) and body condition score (BCS) were recorded at days 0 (T0), 7 (T1), 14 (T2), 21 (T3), 28 (T4) and 35 (T5), according to the American Animal Hospital Association (AAHA) Nutritional Assessment Guidelines for Dogs and Cats [2]. The BW of each animal was measured by the same person at the same time (morning, before feed administration), with the same instrument. At the same time, BCS assessment was carried out by visual examination and palpation of the animal on a scale between 1 and 9 (Figure 1) where a score of 4 or 5 is reflecting the ideal body condition depending on the breed (Body-Condition-Score-Dog.pdf (wsava.org)). To evaluate the effect of the probiotic on fecal quality, an assessment of Fecal Score (FS) and fecal moisture (FM) was performed. Furthermore, some GI bacterial species were identified, and their species count was investigated. Fecal score was evaluated using a 7-point scoring chart according to Bybee and colleagues, as described in Table 5, at all six sampling times (T0-T5).

Table 5: Fecal scoring chart by Nestle Purina fecal score system (modified).

\begin{tabular}{|c|c|}
\hline Score & Characteristics \\
\hline 1 & $\begin{array}{l}\text { Very hard and dry; } \\
\text { Often expelled as individual pellets; } \\
\text { Requires much effort to expel from the body; } \\
\text { Leaves no residue on ground when picked up. }\end{array}$ \\
\hline 2 & $\begin{array}{c}\text { Firm, but not hard, pliable; } \\
\text { Segmented in appearance; } \\
\text { Little or no residue on ground when picked up. }\end{array}$ \\
\hline 3 & $\begin{array}{l}\text { Log-shaped, moist surface; } \\
\text { Little or no visible segmentation; } \\
\text { Leaves residue on ground, but holds form when picked up. }\end{array}$ \\
\hline 4 & $\begin{array}{l}\text { Very moist and soggy; } \\
\text { Log-shaped; } \\
\text { Leaves residue on ground and loses form when picked up. }\end{array}$ \\
\hline 5 & $\begin{array}{l}\text { Very moist but has a distinct shape; } \\
\text { Present in piles rather than logs; } \\
\text { Leaves residue on ground and loses form when picked up. }\end{array}$ \\
\hline 6 & $\begin{array}{l}\text { Has texture, but no defined shape; } \\
\text { Present as piles or spots; } \\
\text { Leaves residue on ground when picked up. }\end{array}$ \\
\hline 7 & $\begin{array}{c}\text { Watery; } \\
\text { No texture; } \\
\text { Present in flat puddles. }\end{array}$ \\
\hline
\end{tabular}

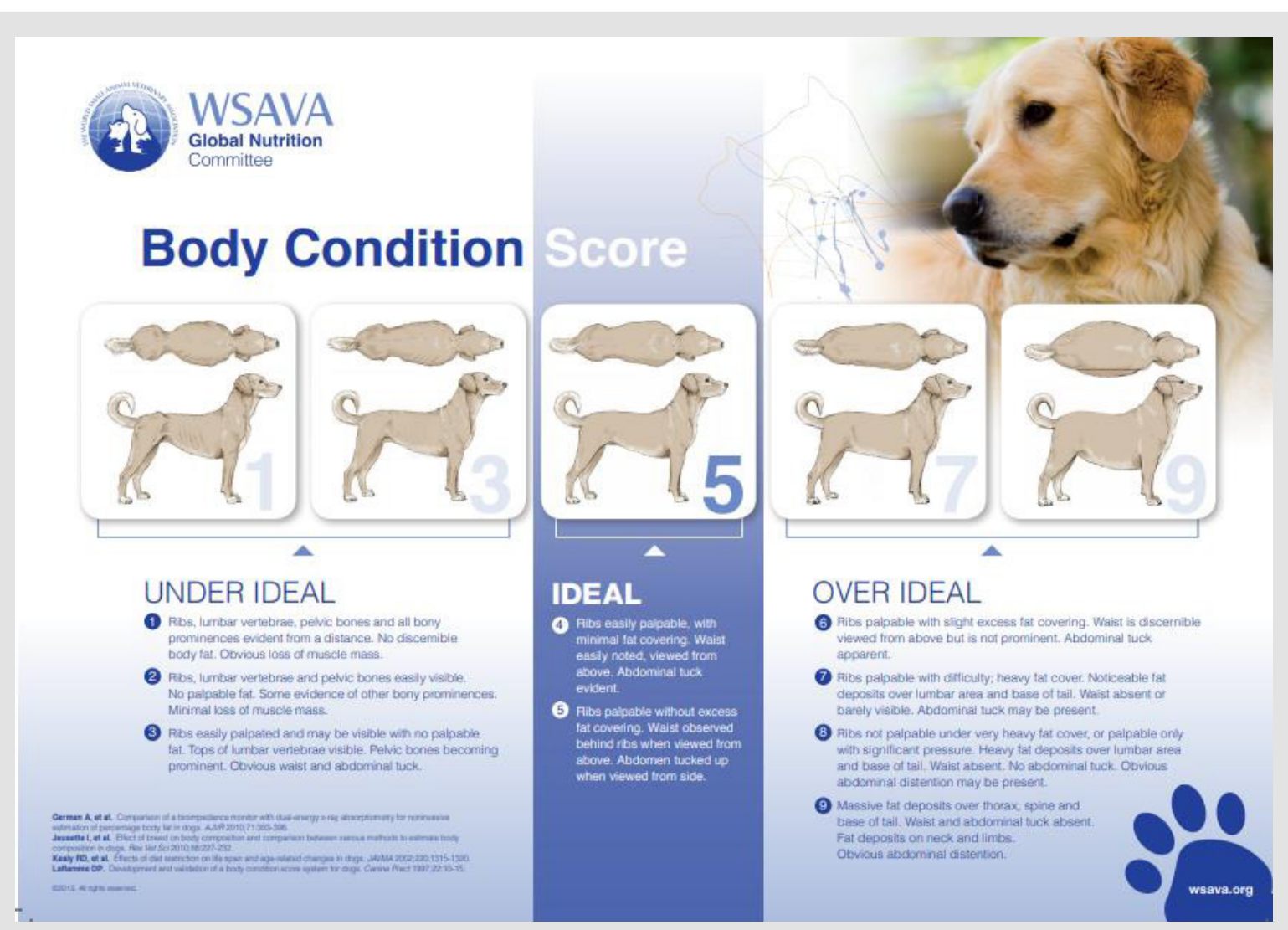

Figure 1: Body Condition Scoring by WSAVA guidelines. 
In the laboratory, collected fecal samples were analyzed to determine FM. Fecal sampling was carried out at T0, T1, T2, T3, T4 and $\mathrm{T} 5$ and the collected samples were stored at $+4^{\circ} \mathrm{C}$ until they are brought to the laboratory, where they are stored at $-20{ }^{\circ} \mathrm{C}$. An aliquot of $5-10 \mathrm{~g}$ of stool was weighed and dried in an oven at a temperature of $105^{\circ} \mathrm{C}-110^{\circ} \mathrm{C}$ for $20-24$ hours, cooled down in a desiccator for another 20-24 hours, after which the FM content was calculated as lost weight after desiccation. Microbiological analysis was performed at T0, T1, T3 and T5. One gram of fresh stool was diluted in sterile saline solution with a ratio of 1:10. Diluted feces were vortexed for two minutes to obtain a homogeneous suspension, which was then streaked on different culture media for total bacterial count and for bacterial identification. Specifically, for Escherichia coli and total coliforms (E. Coli), eosin methylene blue agar (Oxoid, Italy) was used. After an incubation time of 24 hours at $37^{\circ} \mathrm{C}, \mathrm{E}$. coli colonies show growth with a green metallic reflex, while coliforms show growth with blue, red or uncolored colonies. For Lactobacilli, Man Rogosa and Sharpe agar (Oxoid) was used and plates were incubated under anaerobic condition at $37^{\circ} \mathrm{C}$ for 48 hours. All the analysis was performed in duplicate.

\section{Statistical Analysis}

Unlike cats that can be considered 'narrow carnivores', dogs can be called 'optional carnivores' because they have all the characteristics that define carnivores, both anatomically and physiologically, but they are also able to digest and assimilate nutrients such as carbohydrates present in foods such as cereals, legumes and fruits. It's known that carnivorous animals have a different intestinal microflora from herbivores and omnivores; this intestinal microflora serves, among other things, to ferment certain foods, such as carbohydrates. In dogs, the ability to ferment carbohydrates is poor, although it varies depending on the breeds. In fact, there are breeds that assimilate certain nutrients well while others fail to assimilate them at all [3]. However, the source of carbohydrates would not seem to be indispensable for dog as it has alternative metabolic processes through which it produces glucose from proteins, so, according to the nutritional epigenetics, during the process of domestication of the dog, environmental pressures may have caused a change in the synthesis of enzymes in charge of digestion of nutrients, so that dogs adapted to live on a diet based on the waste of the human diet; this would explain why nowadays dogs can assimilate numerous substances of plant origin [4].

For this reason, the dog feed that is on the market provides complete and balanced nutrition also presenting a fairly high content of carbohydrates and fiber; this food component is very important because several studies have shown that fiber is fermented by specific beneficial bacteria present at the intestinal level by promoting an increase in short-chain fatty acids that have countless beneficial effects for the well-being of the animal by decreasing the intestinal $\mathrm{pH}$ and also competing with pathogenic bacteria for adhesion sites and nutrient. So, the type of diet is able to modulate the composition of the intestinal microbiota by influencing the number and species of micro-organisms present, especially as a function of quantity and quality of fiber present in the food [5]. The dietary modulation of the intestinal microbiota also has direct consequences on the quality and quantity of fecal material [6] as the microorganisms present above all in the last intestinal tract of the dog are able to ferment the undigested material with consequences both on the intestinal environment and on the feces excreted by the animal [7].

Among the different feeding strategies that can be used to modulate the intestinal microbiota of the dog, in the bibliography we find the use of lactic bacteria [8]. On the other hand, the intestinal microbiota needs more time to show its action on the quality of feces compared to sudden dietary changes. For the above reasons to evaluate the effects of $L$. reuteri NBF 1 DSM 32203 on the parameter relating to fecal quality in dogs, for the calculation of the sample number for each group was applied the classic formula:

$$
n>2\left[(z \alpha / 2+z \beta)_{*} \sigma / \delta\right]^{2}
$$

where $\sigma$ represents the standard deviation, derived from pilot studies or literature, while $\delta(\mu 1-\mu 2)$ is the minimum clinically relevant difference. As regards the identification of $\sigma$ and $\delta$, we also used the data of a previous study [9], considering the variable of the fecal score. Considering that the dogs included in this study are healthy (without any gastrointestinal pathology) and living in controlled breeding conditions (without any variation in the daily food ration), the expected variability will be low and therefore a variation of 0.4 points in the fecal score can be considered biologically relevant with a standard deviation of 0.512 .

Applying the above values to the above formula we will have:

$$
2[(1.64+0.84) * 1.28]^{2}=20.15 \sim 20
$$

Then the formula used for the calculation of the sample size gives us the number of 20 dogs for each experimental group as the minimum number of animals that must complete the study period to detect a difference of 0.4 points in the fecal score between the groups with an alpha of 0.1 and a beta of 0.8. For the statistical analysis a Mixed Model with repeated measurements has been used, which allows to estimate the parameters considering both random effect and fixed effect. Following [10], the model has been estimated as the following:

$$
Y_{i, j, k}=\mu+S_{i}+G_{j}+T_{k}+G_{j} * T_{k}+e_{i, j, k}
$$

where $\mathrm{y}=$ dependent variable (FM, FS, BW, BCS, LB, COLI); $\mu=$ overall mean; $\mathrm{Si}=$ fixed effect of the ith $\operatorname{sex}(I=1,2) ; \mathrm{G}_{\mathrm{j}}=$ fixed effect of the jth group $(j=1,2) ; T_{k}=$ fixed effect of the kth time $(k=0.5)$ and $e_{i, j, k}=$ error. The software used was: R Core Team (2020), R: A language and environment for statistical computing ( $\mathrm{R}$ Foundation 
for Statistical Computing, Vienna, Austria) and for the different analysis was used the Mixed Model (Pinheiro J, Bates D, DebRoy S, Sarkar D, R Core Team (2019); nlme: Linear and Nonlinear Mixed Effects Models. R package version 3.1-141) and the Least Squares [11], Least-Squares Means: The R Package lsmeans (Journal of Statistical Software, 69(1), 1-33. doi:10.18637/jss.v069.i01). Time was used as repeated measurement and therefore each subject has been analyzed in every different temporal instant. The autoregressive covariance structure was used. Least Square Means were estimated and they were been statistically tested using Student's t test (with Tukey p-value adjustment). In order to be able to describe the goodness of the fit of the mixed model, we used the R squared described by [12], No outliers and missing data were found.

\section{Results}

Table 6: Effect of Lactobacillus reuteri NBF 1 DSM 32203 addition to diet on body weight (BW) (A) and body condition score (BCS) (B) of Golden Retriever dogs.

Table 6A: Effect of Lactobacillus reuteri addition to diet on body weight (BW) of Golden Retriever dogs (LS Mean \pm SE).

\begin{tabular}{|c|c|c|c|}
\hline \multicolumn{4}{|c|}{ Groups } \\
\hline Time & \multicolumn{1}{|c|}{ CTR } & LACT0 & P- value \\
\hline Overall & $31.3 \pm 0.4$ & $31.2 \pm 0.4$ & 0.523 \\
\hline T0 & $31.3 \pm 0.5$ & $31.2 \pm 0.5$ & 0.999 \\
\hline T1 & $31.3 \pm 0.5$ & $31.2 \pm 0.5$ & 1 \\
\hline T2 & $31.3 \pm 0.5$ & $31.2 \pm 0.5$ & 1 \\
\hline T3 & $31.3 \pm 0.5$ & $31.2 \pm 0.5$ & 0.997 \\
\hline T4 & $31.3 \pm 0.5$ & $31.2 \pm 0.5$ & 0.998 \\
\hline T5 & $31.3 \pm 0.5$ & $31.2 \pm 0.5$ & 1 \\
\hline
\end{tabular}

Table 6B: Effect of Lactobacillus reuteri addition to diet on body condition score (BCS) of Golden Retriever dogs (LS Mean \pm SE)

\begin{tabular}{|c|c|c|c|}
\hline \multicolumn{4}{|c|}{ Groups } \\
\hline Time & CTR & LACT0 & P-value \\
\hline Overall & $4.73 \pm 0.11$ & $4.75 \pm 0.11$ & 0.719 \\
\hline T0 & $4.73 \pm 0.22$ & $4.77 \pm 0.22$ & 1 \\
\hline T1 & $4.73 \pm 0.22$ & $4.75 \pm 0.22$ & 1 \\
\hline T2 & $4.75 \pm 0.22$ & $4.75 \pm 0.22$ & 1 \\
\hline T3 & $4.70 \pm 0.22$ & $4.77 \pm 0.22$ & 1 \\
\hline T4 & $4.73 \pm 0.22$ & $4.72 \pm 0.22$ & 1 \\
\hline T5 & $4.73 \pm 0.22$ & $4.75 \pm 0.22$ & 1 \\
\hline
\end{tabular}

All dogs were healthy during the trial, no side effects and no case of death were recorded as evidenced by the certificate issued by the veterinarian. No residual pet food was found after consumption throughout the experimental period. BW and BCS did not change during the trial in either group showing no significant values (Tables 6A \& 6B) and the animals maintained an ideal body condition. BW data show no differences between the two groups. FM was significantly lower at the end of the trial in the LACTO group compared with the CTR group $(\mathrm{P}<0.001$; Table 7$)$ in fact a lower humidity content was found especially in the last three weeks of the experimental period (T3-T5) in the fecal samples of the group treated with L. reuteri NBF 1 DSM 32203 compared with the values recorded in the CTR group $(\mathrm{P}<0.001)$ where the fecal humidity remains almost the same; the beneficial effect of Lactobacillus reuteri NBF 1 DSM 32203 was also confirmed by the values of fecal score (FS) recorded among the two groups of dogs (Table 8) (Figure 2). Specifically, FS values fluctuate between $3.34 \pm 0.19$ at the beginning of the study and $3.31 \pm 0.19$ at the end in the control group, so, there wasn't any variation; while, the LACTO group shows much lower values between the $3.38 \pm 0.19$ at the beginning of the trial and the $2.08 \pm 0.19$ at the end of the experiment with an overall value of $3.34 \pm 0.10(\mathrm{P}<0.001)$ for the control group and $2.63 \pm 0.10$ $(\mathrm{P}<0.001)$ for the LACTO group.

Table 7: Effect of Lactobacillus reuteri NBF 1 DSM 32203 addition to diet on fecal moisture in dogs: results of mixed models showing least square means \pm SE in CTR (control group) and LACTO (treated group) dogs for the six individual sampling times and overall throughout the study.

\begin{tabular}{|c|c|c|c|}
\hline Time & CTR & LACT0 & P-value \\
\hline Overall & $0.71 \pm 0.01$ & $0.61 \pm 0.01$ & $<0.001$ \\
\hline T0 & $0.71 \pm 0.02$ & $0.71 \pm 0.02$ & 1 \\
\hline T1 & $0.71 \pm 0.02$ & $0.68 \pm 0.02$ & 0.003 \\
\hline T2 & $0.71 \pm 0.02$ & $0.64 \pm 0.02$ & $<0.001$ \\
\hline T3 & $0.71 \pm 0.02$ & $0.59 \pm 0.02$ & $<0.001$ \\
\hline T4 & $0.71 \pm 0.02$ & $0.54 \pm 0.02$ & $<0.001$ \\
\hline T5 & $0.70 \pm 0.02$ & $0.50 \pm 0.02$ & $<0.001$ \\
\hline
\end{tabular}

Table 8: Effect of Lactobacillus reuteri NBF 1 DSM 32203 addition to diet on the fecal score of Golden Retriever adult healthy dogs: results of mixed models showing least square means \pm SE in CTR (control group) and LACTO (treated group) dogs for the six individual sampling times and overall throughout the study.

\begin{tabular}{|c|c|c|c|}
\hline Time & CTR & LACT0 & P-value \\
\hline Overall & $3.34 \pm 0.10$ & $2.63 \pm 0.10$ & $<0.001$ \\
\hline T0 & $3.34 \pm 0.19$ & $3.38 \pm 0.19$ & 1 \\
\hline T1 & $3.39 \pm 0.19$ & $3.15 \pm 0.19$ & 0.265 \\
\hline T2 & $3.34 \pm 0.19)$ & $2.73 \pm 0.19$ & $<0.001$ \\
\hline T3 & $3.31 \pm 0.19$ & $2.33 \pm 0.19$ & $<0.001$ \\
\hline T4 & $3.36 \pm 0.19$ & $2.13 \pm 0.19$ & $<0.001$ \\
\hline T5 & $3.31 \pm 0.19$ & $2.08 \pm 0.19$ & $<0.001$ \\
\hline
\end{tabular}


FS boxplots for groups - Time 0

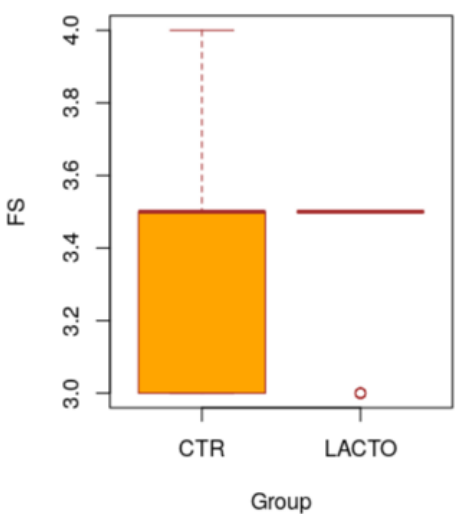

FS boxplots for groups - Time 5

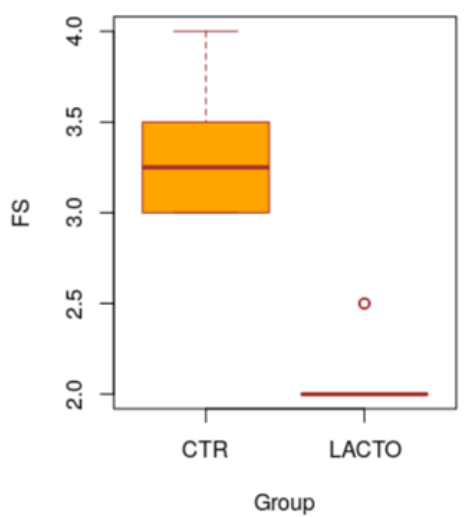

Figure 2: Box plot showing the effect of Lactobacillus reuteri NBF 1 DSM 32203 addition to the diet on the fecal score (FS) of Golden Retriever dogs in the overall period ( $\mathrm{P}<0.001$; t-test). CTR, control group; LACTO, treated group.

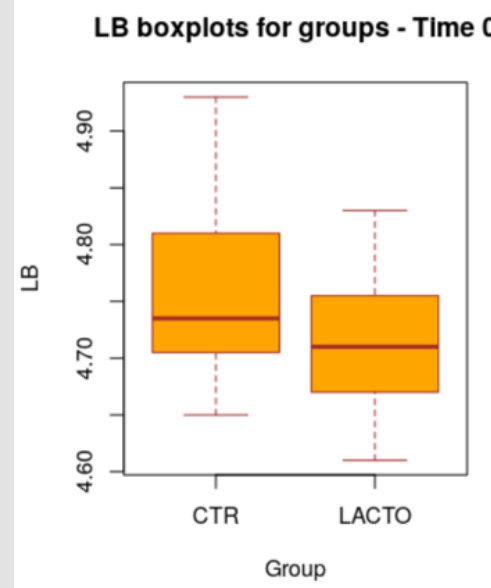

\section{LB boxplots for groups - Time 5}

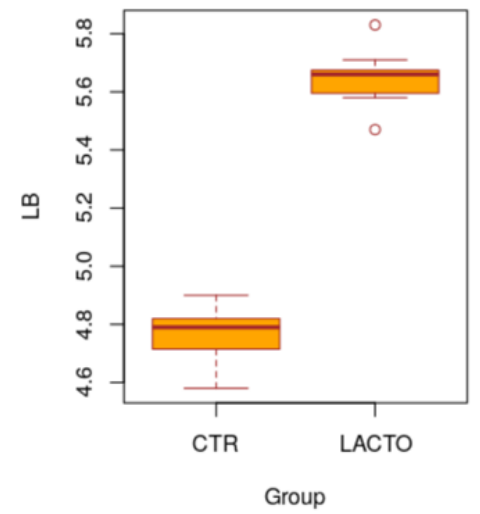

Figure 3: Box plot showing the effect of Lactobacillus reuteri NBF 1 DSM 32203 addition to diet on total Lactobacilli count (LB) in the overall period $(\mathrm{P}<0.001$; t-test). CTR, control group; $\mathrm{LACTO}$, treated group.

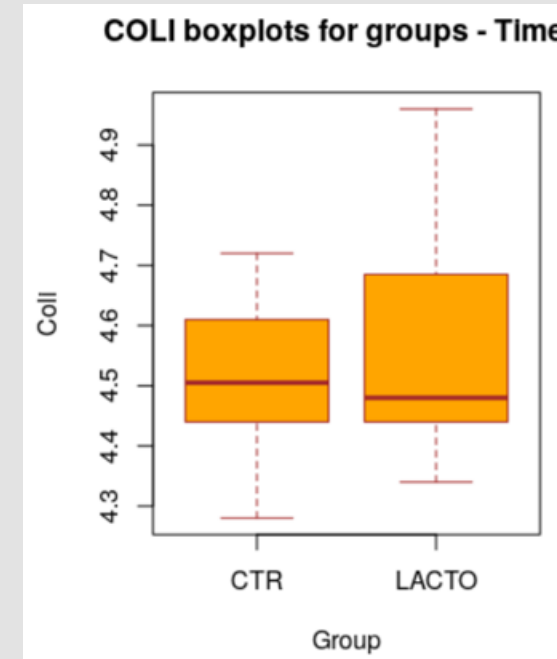

COLI boxplots for groups - Time 5

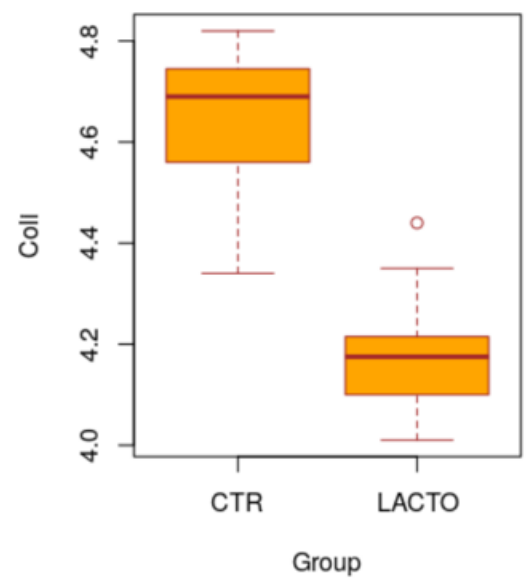

Figure 4: Box plot showing the effect of Lactobacillus reuteri NBF 1 DSM 32203 addition to diet on total coliform (Coli) in the overall period $(\mathrm{P}<0.001$; t-test). CTR, control group; LACTO, treated group. 
The decrease of about 1.2 points (registered in the LACTO group) on a scale of 1 to 7 can certainly have implications for the intestinal health of dogs with an important biological relevance [13], With regard to the microbiological analysis, at the end of the experimental period (T5), was observed a significant increase of Lactobacilli in the treated group (LACTO) respect to the control group moving from a concentration of $4.72 \pm 0.05 \mathrm{log} \mathrm{CFU} / \mathrm{g}$ $(\mathrm{P}=0.891)$ at the beginning of the experiment to a concentration of $5.65 \pm 0.05 \log C F U / g(P<0.001)$ at the end of the trial (Table 9) (Figure 3) followed by a little decrease in the total coliforms' amount $(\mathrm{P}<0.001)$ (Table 10) (Figure 4).

Table 9: Effect of Lactobacillus reuteri NBF 1 DSM 32203 addition to diet, expressed as $\log \mathrm{CFU} / \mathrm{g}$, on the total amount of Lactobacilli present in the intestinal microflora of Golden Retriever adult healthy dogs: results of mixed models showing least square means \pm SE in CTR (control group) and LACTO (treated group) dogs for the four individual sampling times and overall throughout the study.

\begin{tabular}{|c|c|c|c|}
\hline Time & CTR & LACT0 & P-value \\
\hline Overall & $4.75 \pm 0.03$ & $5.12 \pm 0.03$ & $<0.001$ \\
\hline T0 & $4.75 \pm 0.05$ & $4.72 \pm 0.05$ & 0.891 \\
\hline T1 & $4.73 \pm 0.05$ & $4.92 \pm 0.05$ & $<0.001$ \\
\hline T3 & $4.75 \pm 0.05$ & $5.20 \pm 0.05$ & $<0.001$ \\
\hline T5 & $4.76 \pm 0.05$ & $5.65 \pm 0.05$ & $<0.001$ \\
\hline
\end{tabular}

Table 10: Effect of Lactobacillus reuteri NBF 1 DSM 32203 addition to diet, expressed as $\log \mathrm{CFU} / \mathrm{g}$, on the amount of E. coli present in the intestinal microflora of Golden Retriever adult healthy dogs: results of mixed models showing least square means \pm SE in CTR (control group) and LACTO (treated group) dogs for the four individual sampling times and overall, throughout the study.

\begin{tabular}{|c|c|c|c|}
\hline Time & CTR & LACT0 & P-value \\
\hline Overall & $4.58 \pm 0.05$ & $4.39 \pm 0.05$ & $<0.001$ \\
\hline T0 & $4.51 \pm 0.08$ & $4.55 \pm 0.08$ & 0.956 \\
\hline T1 & $4.54 \pm 0.08$ & $4.48 \pm 0.08$ & 0.774 \\
\hline T3 & $4.63 \pm 0.08$ & $4.34 \pm 0.08$ & $<0.001$ \\
\hline T5 & $4.64 \pm 0.08$ & $4.19 \pm 0.08$ & $<0.001$ \\
\hline
\end{tabular}

\section{Discussion}

In this study were analyzed the effects of a specific probiotic: L. reuteri NBF 1 DSM 32203 on body weight, fecal consistency and microbiological analysis of fecal samples of healthy adult dogs. During the experimental period there was a significant reduction in fecal humidity (FM) and the fecal score (FS) registered in the group of dogs treated with the probiotic (LACTO) displayed a mean value closer to the ideal compared with the control group (CTR). These data suggest a positive effect of the L. reuteri NBF 1 DSM 32203 at the intestinal level of healthy dogs decreasing fecal humidity giving more consistency to the stool. The lowest fecal humidity values were recorded in the LACTO group during the last three weeks of study (T3, T4 and T5); even with regard to the fecal score, in the last three weeks of study (T3-T5) in the LACTO group were recorded scores closer to those of the 'ideal' condition respect to the control group (CTR).

The effects of the administration of L. reuteri NBF 1 DSM 32203 on the intestinal microbial ecosystem showed the ability of this probiotic to increase, especially in the last two weeks, the amounts of lactobacilli, followed by a little decrease of total coliforms. The increase in lactobacilli is certainly positive: they promote the integrity of the intestinal barrier by preventing the adhesion of pathogenic bacteria and subsequent their proliferation [14].

\section{Conclusion}

The data collected in this study report the ability of the probiotic L. reuteri NBF 1 DSM 32203 to improve fecal quality parameters such as FM and FS in healthy adult dogs. One of the most important parameters at the level of biological relevance is that of fecal score that at the end of the treatment reported a significant decrease of about 1.2 points in the group treated with L. reuteri NBF 1 DSM 32203 compared to the control group that showed no decrease respect the beginning. This result was accompanied by a decrease in fecal humidity, causing the feces to be more consistent, hard and well-formed as an indication of good intestinal health related to a good digestion [15]. In addition, the increase in Lactobacilli, confirms once again the ability of this probiotic to improve the composition of the intestinal ecosystem by promoting an increase in beneficial species capable of promoting the maintenance of the integrity of the intestinal mucosa.

\section{References}

1. (2018) FEDIAF: Nutritional guidelines. (FEDIAF_Nutritional_Guidelines.

2. Baldwin K, Bartges J, Buffington T (2010) AAHA nutritional assessment guidelines for dogs and cats. J Am Anim Hosp Assoc 46(4): 285-296.

3. Weber MP, Biourge VC, Nguyen PG (2017) Digestive sensitivity varies according to size of dogs: a review. Journal of animal physiology and animal nutrition 101(1): 1-9.

4. Landecker H (2011) Food as exposure: Nutritional epigenetics and the new metabolism. BioSocieties 6(2): 167-194.

5. Gzerskowiak L, Endo A, Beasley S, Salminen S (2015) Microbiota and probiotics in canine and feline welfare. Anaerobe 34: 14-23.

6. Huang Z, Pan Z, Yang R, Bi Y, Xiong X (2020) The canine gastrointestinal microbiota: early studies and research frontiers 11(4): 635-654.

7. Rose C, Parker A, Jefferson B, Cartmell E (2015) The characterization of feces and urine: a review of the literature to inform advanced treatment technology. Critical Reviews in Environmental Science and Technology 45(17): 1827-1879.

8. Jensen AP, Bjørnvad CR (2019) Clinical effect of probiotics in prevention or treatment of gastrointestinal disease in dogs: A systematic review. J Vet Intern Med 33(5): 1849-1864. 
9. Stokes JE, Price JM, Whittemore JC (2017) Randomized, controlled, crossover trial of prevention of clindamycin-induced gastrointestinal signs using a synbiotic in healthy research cats. J Vet Intern Med 31(5) 1406-1413.

10. Fusi E, Rizzi R, Polli M (2019) Effects of Lactobacillus acidophilus D2/CSL (CECT 4529) supplementation on healthy cat performance. Veterinary Record Open 6: e000368.

11. Means Russell V Lent (2016) Least-Squares Means: The R Package lsmeans. Journal of Statistical Software 69(1): 1-33.

12. Nakagawa S, Schielzeth H (2013) A general and simple method for obtaining R2 from generalized linear mixed-effects models. Methods Ecol Evol 4(2): 133-142.

ISSN: 2574-1241

DOI: 10.26717/BJSTR.2021.37.005956

Benedetta Belà. Biomed J Sci \& Tech Res

(C) (P) This work is licensed under Creative

Submission Link: https://biomedres.us/submit-manuscript.php
13. Detweiler KB, He F, Mangian HF, Davenport GM, De Godoy MRC (2019) Effects of high inclusion of soybean hulls on apparent total tract macronutrient digestibility, fecal quality, and fecal fermentative endproduct concentrations in extruded diets of adult dogs. J Anim Sci 97(3): 1027-1035.

14. Cazorla SI, Maldonado Galdeano C, Weill R, De Paula J, Perdigon GDV (2018) Oral administration of probiotics increases Paneth cells and intestinal antimicrobial activity. Frontiers in microbiology, pp. 736

15. Ohno H, Murakami H, Tanisawa K, Konishi K, Miyachi M (2019) Validity of an observational assessment tool for multifaceted evaluation of faecal condition. Scientific Reports 9: 3760. 